\title{
The antioxidant effect of $\beta$-caryophyllene protects rat liver from carbon tetrachloride-induced fibrosis by inhibiting hepatic stellate cell activation
}

\author{
Miguel Angel Calleja ${ }^{1}$, Jose María Vieites ${ }^{2}$, Trinidad Montero-Meterdez ${ }^{3}$, María Isabel Torres ${ }^{4}$, María \\ José Faus ${ }^{5}$, Angel Gil ${ }^{5}$ and Antonio Suárez ${ }^{6 *}$ \\ ${ }^{1}$ UGC Farmacia, Hospital Universitario Virgen de las Nieves, Granada, Spain \\ ${ }^{2}$ Bioquímica de Alimentos, Instituto de Investigaciones Marinas (CSIC), Vigo, Spain \\ ${ }^{3}$ Biochemical Pharmacology, William Harvey Research Institute, Queen Mary University of London, London, UK \\ ${ }^{4}$ Departamento de Biología Experimental, Universidad de Jaen, Jaen, Spain \\ ${ }^{5}$ Departamento de Bioquímica y Biología Molecular 2, Facultad de Farmacia, Universidad de Granada, Granada, Spain \\ ${ }^{6}$ Instituto de Nutrición y Tecnología de los Alimentos, Centro de Investigación Biomédica, Armilla, Spain
}

(Submitted 1 April 2011 - Final revision received 20 February 2012 - Accepted 27 February 2012 - First published online 1 May 2012)

\section{Abstract}

Plant-based whole foods provide thousands of bioactive metabolites to the human diet that reduce the risk of developing chronic diseases. $\beta$-Caryophyllene (CAR) is a common constituent of the essential oil of numerous plants, vegetables, fruits and medicinal herbs, and has been used as a flavouring agent since the $1930 \mathrm{~s}$. Here, we report the antioxidant activity of CAR, its protective effect on liver fibrosis and its inhibitory capacity on hepatic stellate cell (HSC) activation. CAR was tested for the inhibition of lipid peroxidation and as a free radical scavenger. CAR had higher inhibitory capacity on lipid peroxidation than probucol, $\alpha$-humulene and $\alpha$-tocopherol. Also, CAR showed high scavenging activities against hydroxyl radical and superoxide anion. The activity of 5-lipoxygenase, an enzyme that actively participates in fibrogenesis, was significantly inhibited by CAR. Carbon tetrachloride-treated rats received CAR at 2,20 and $200 \mathrm{mg} / \mathrm{kg}$. CAR significantly improved liver structure, and reduced fibrosis and the expression of Col1a1, Tgfb1 and Timp1 genes. Oxidative stress was used to establish a model of HSC activation with overproduction of extracellular matrix proteins. CAR ( 1 and $10 \mu \mathrm{M})$ increased cell viability and significantly reduced the expression of fibrotic marker genes. CAR, a sesquiterpene present in numerous plants and foods, is as a natural antioxidant that reduces carbon tetrachloride-mediated liver fibrosis and inhibits hepatic cell activation.

\section{Key words: $\beta$-Caryophyllene: Liver fibrosis: Antioxidants: Hepatic stellate cells}

Epidemiological studies have consistently shown that regular consumption of fruits and vegetables as well as whole grains is strongly associated with a reduced risk of developing chronic diseases, such as cancer and CVD, which are the top two causes of death in most industrialised countries ${ }^{(1)}$. These studies highlighted the unique chemical features of fruits, vegetables, spices and grains. Plant-based whole foods provide thousands of primary and secondary metabolites to the human diet that are absorbed into the body due to their low-molecular lipophilic nature. These phytochemicals are bioactive non-nutrient plant compounds that can be classified into various families, such as alkaloids, flavonoids, isoflavones, isothiocyanates, organo-sulphur compounds, capsaicinoids, phytosterols, carotenoids and terpenoids ${ }^{(2)}$.
Particularly, terpenoids are perhaps the most diverse family of natural products synthesised from plants, serving a range of important physiological functions. Terpenoids are the major volatile constituents of essential oils that generate the 'flavour fingerprint' of vegetables, fruits and spices ${ }^{(3)}$. The essential oils of plants, fruits and vegetables are natural, complex, multicomponent systems composed mainly of terpenes in addition to some other non-terpene components. Formed via the isoprene pathway, these compounds are grouped into hydrocarbons (limonene), alcohols (menthol), aldehydes (citral), ketones (carvone), acids and esters (geranyl acetate). Since this pathway operates to a different extent in plants, many of the same chemical constituents are present in a wide variety of essential oils. A wide range of terpenoids has demonstrated

Abbreviations: ALAT, alanine aminotransferase; ASAT, aspartate aminotransferase; CAR, $\beta$-caryophyllene; Col1a1, type I collagen $\alpha 1$; DPPH, 1 , 1 -diphenyl2-picrylhydrazyl; HSC, hepatic stellate cell; LDH, lactate dehydrogenase; TBARS, thiobarbituric acid-reactive substances; TGF- $\beta 1$, transforming growth factor- $\beta 1$; Timp1, tissue inhibitor of metalloproteinase-1.

*Corresponding author: A. Suárez, fax +34958 819132, email asuarez@ugr.es 
pharmaceutical activity against human ailments such as inflammatory diseases ${ }^{(4)}, \mathrm{CVD}^{(5)}$ and cancer ${ }^{(6)}$.

Our group previously reported the hepatoprotective activity of an ethanol extract of Rosmarinus tomentosus Huberth-Morat and Maire (Lamiaceae) in $\mathrm{CCl}_{4}^{-}$and thioacetamide-induced cirrhotic rats ${ }^{(7,8)}$. Because reactive oxygen species are generated during $\mathrm{CCl}_{4}$-induced liver toxicity, we explored the free-radical-scavenging capacity of the most frequent terpenes in Rosmarinus spp. essential oil. The ethanol extract in Rosmarinus officinalis contains a variable mixture of phenolic diterpenes (carnosic acid, carnosol and 12-O-methylcarnosic acid), caffeoyl derivatives (rosmarinic acid) and flavones with other terpenes in lower quantities ${ }^{(9)}$. The present study contributes extensive data on the identification of $\beta$-caryophyllene (CAR) as a free-radical-scavenging agent and on its capacity to inhibit lipid peroxidation in vitro. CAR protected rat livers from $\mathrm{CCl}_{4}$ toxicity and reduced fibrosis and the gene expression of type I collagen $\alpha 1$ (Col1a1), transforming growth factor- $\beta 1$ (Tgfb1) and tissue inhibitor of metalloproteinase-1 (Timp1). Ex vivo, CAR protected CFCS$2 \mathrm{G}$ cells, a rat hepatic stellate cell (HSC) line, from free radical damage and effectively down-regulated the expression of the Col1a1 and Timp1 genes. CAR is a volatile sesquiterpene found in large amounts in the essential oils of many herbal drugs, spice and food plants, including oregano, cinnamon, black pepper, carrot, bay laurel, grapes, melon, red blush grapefruit, strawberry, guava, ginger, among others. The present results demonstrated that CAR is an antifibrogenic candidate for the prevention and treatment of hepatic fibrosis. Since patients with liver fibrosis have an associated condition of malnutrition, our data support the need to increase the consumption of fruits, vegetables and whole grains as a source of bioactive non-nutrient natural compounds to maintain a healthy liver status.

\section{Experimental methods}

\section{Chemicals}

All chemicals (unless specified) were obtained from SigmaAldrich Química S.A., at the highest available grades.

\section{In vitro cell culture}

The CFSC-2G cell line has a similar phenotype to that of early-passage primary HSC ${ }^{(10)}$. CFSC-2G cells were maintained in culture with minimum essential medium supplemented with $10 \%$ fetal bovine serum, $0 \cdot 1 \%$ non-essential amino acids and $1.1 \%$ penicillin/streptomycin. For each experiment, cells were incubated overnight with no additions and then treated with appropriate concentrations of CAR at $24 \mathrm{~h}$ before treatment with an $\mathrm{Fe}^{2+}$-ascorbate solution (final concentration 50$200 \mu \mathrm{M}$ ). Culture medium and cells were collected $6 \mathrm{~h}$ later.

\section{Animals and treatments}

Female Wistar rats $(150-170 \mathrm{~g})$ were housed in cages at constant temperature $\left(22^{\circ} \mathrm{C}\right)$ and humidity $(55 \%)$ with a $12 \mathrm{~h}$ light-12h dark cycle (06.00-18.00 hours). The animals were handled in accordance with recommendations of the American Physiological Society (Council of Europe, 1982). The animals were randomly divided into five groups of six individuals with free access to water and rodent chow. To produce acute liver damage, the experimental design consisted in a single $\mathrm{CCl}_{4}(0 \cdot 15 \mathrm{ml} / 100 \mathrm{~g}$ body weight $)$ intraperitoneal injection. Dose selection for CAR treatment was based on previously published studies ${ }^{(11)}$. Similarly to epigallocathechin-3gallate treatment, CAR administration was started in animals subjected to acute $\mathrm{CCl}_{4}$ on the day before $\mathrm{CCl}_{4}$ poisoning ${ }^{(12)}$. Body weight was monitored throughout the experiment. Rats were deprived of food for $12 \mathrm{~h}$, anaesthetised and blood was collected from the abdominal aorta. The liver was removed, washed with cold saline, weighed and stored at $-80^{\circ} \mathrm{C}$.

\section{Liver histology and collagen content}

A portion of the liver was fixed in 10\% formaldehyde, embedded in paraffin and cut into $5 \mathrm{~mm}$-thick sections. The samples were stained with periodic acid-Schiff and fast green-neutral red. A semi-quantitative score for hepatic injury ranging from 0 to 5 was assigned to each rat according to the presence or absence of hepatocyte necrosis, nodular transformation, fibrous septae and biliary hyperplasia. Fibrosis severity was scored as normal (0), minimal (1), mild (2), moderate (3), marked (4) or severe fibrosis (5). Collagen content was measured in $15 \mu \mathrm{m}$-thick liver sections using a colorimetric method based on the selective capacity of two dyes (Sirius red F3B and fast green FCF) to bind to collagen and non-collagenous proteins, respectively, in the presence of picric $\operatorname{acid}^{(13)}$.

\section{Measurement of free radical-scavenging activity}

Radical-scavenging activity against the 1,1-diphenyl-2-picrylhydrazyl (DPPH) radical was determined by using the method of Mensor $e t a l .{ }^{(14)}$. Scavenging activity against the hydroxyl radical was determined by the inhibition of chemiluminescence during linoleic acid peroxidation by the Fenton reaction, as described by Xun et al. ${ }^{(15)}$. Scavenging activity against the superoxide anion was assayed in non-enzymatic and enzymatic systems. First, the compounds were tested to inhibit the photochemical oxidation of $o$-dianisidine by riboflavin, as described by Bridges et $a l .{ }^{(16)}$. Second, the enzymatic production of the superoxide anion was assayed by monitoring the reduction in cytochrome $c^{(17)}$. The inhibition percentage of superoxide anion generation was calculated according to the formula: percentage inhibition $=\left(A_{\text {control }}-A_{\text {sample }}\right) / A_{\text {control }} \times 100$. The inhibition of the formation of lipid peroxides by 5 -lipoxygenase was performed as described by Liu \& Pan ${ }^{(18)}$.

\section{Inhibition of lipid peroxidation}

Liver microsomes were obtained as described by Suárez et $a l .{ }^{(19)}$. Lipid peroxidation was induced in vitro in liver microsomes with a $\mathrm{Fe}^{2+}$-ascorbate solution $(50-200 \mu \mathrm{M})$. The test compounds were added to liver microsomes 
30 min before initiating lipid peroxidation. Thiobarbituric acidreactive substances (TBARS) were assayed in the medium at $30 \mathrm{~min}$ after induction and expressed as nmol malondialdehyde/mg $\operatorname{protein}^{(19)}$.

\section{Analytic determinations}

Total bilirubin levels were determined with the standard 2,5-dichlorophenyldiazonium tetrafluoroborate diazo reagent, according to the manufacturer's instructions (Roche Applied Science). Plasma protein content was determined according to the method of Bradford ${ }^{(20)}$. Lactate dehydrogenase (LDH) was spectrophotometrically assayed to test cell viability (Sigma-Aldrich Química S.A.). Aspartate aminotransferase (ASAT), alanine aminotransferase (ALAT), alkaline phosphatase and $\gamma$-glutamyltranspeptidase activities were measured as described previously ${ }^{(7,8)}$. Enzyme units were expressed per mg of plasma protein.

\section{Gene expression}

Total RNA was extracted from the rat liver tissue or CFSC-2G cells. Briefly, $10 \mu \mathrm{g}$ of total RNA were electrophoresed in agarose gels, transferred to nylon filters, fixed with a UV crosslinker and hybridised with an $\alpha-{ }^{32} \mathrm{P}$-deoxycytidine triphosphate-labelled complementary DNA probe specific for Col1a1 or Timp1 mRNA and, sequentially, with an $\alpha-{ }^{32} \mathrm{P}-$ deoxycytidine triphosphate-labelled oligonucleotide complementary to glyceraldehyde-3-phosphate dehydrogenase (Gapdb) mRNA. Semi-quantitative PCR was used to measure Col1a1, Tgfb1 and Timp1 gene expression in the rat liver as described by Pérez et al. ${ }^{(13)}$.

\section{Statistical analysis}

All data are presented as mean values with their standard errors. All variables were checked for normality and homogeneous variance by using the Kolmogorov-Smirnov and Levene tests, respectively. A two-way ANOVA was used to evaluate differences according to compound and concentration, and a posteriori Bonferroni tests were carried out for specific mean comparisons. One-way ANOVA and a post boc mean comparison using the Bonferroni test were used to analyse the effects between CAR, vitamin E and control groups, and to assess concentration effects for specific compounds. A value of $P<0.05$ was considered significant. Data analyses were performed using SPSS for Windows 15.0 (SPSS, Inc.).

\section{Results}

\section{$\beta$-Caryophyllene is a free-radical-scavenging constituent} of Rosmarinus spp. essential oil

Based on published data on essential oil constituents in Rosmarinus spp. ${ }^{(9)}$, we first tested the inhibitory capacity of $\alpha$-pinene, camphene, $\alpha$-humulene, camphor, borneol, 1,8cineole and CAR against liver microsome lipid peroxidation. Only $\alpha$-humulene and CAR showed inhibitory capacity (data not shown), which was further assayed in a concentrationdependent test. At all concentrations, CAR had a significantly higher inhibitory action against lipid peroxidation than did $\alpha$-humulene, probucol and vitamin E (Fig. 1). Probucol and vitamin $\mathrm{E}$ were included as positive controls for synthetic and natural antioxidant compounds. CAR showed scavenging activity against the hydroxyl radical (Fenton reaction) and superoxide anion (Riboflavin assay) in a concentration-dependent manner (Table 1). CAR also inhibited superoxide aniondependent cytochrome $c$ reduction in the hypoxanthine/ xanthine oxidase system (Table 1). CAR inhibited lipoxygenase-dependent oxidation of linoleic acid in a similar manner to $\alpha$-tocopherol. CAR showed greater free-radical-scavenging activity in comparison with probucol and greater superoxide anion- and similar hydroxyl radical-scavenging activities in comparison with $\alpha$-tocopherol.

\section{Carbon tetrachloride-induced liver fibrosis was markedly suppressed by $\beta$-caryophyllene}

A fourth experiment was designed to determine the protective effect of $\mathrm{CAR}$ on $\mathrm{CCl}_{4}$-induced liver injury. Pretreatment with CAR significantly reduced plasma ASAT, ALAT and LDH activities and total bilirubin levels in a dose-dependent manner (Table 2). Representative images of liver microscopic morphology are shown in Fig. 2. Histological analysis of the liver of $\mathrm{CCl}_{4}$-treated rats revealed marked fibrosis with hepatocyte necrosis, inflammatory cell infiltration, fibrosis and microvesicular steatosis. In contrast, CAR significantly reduced the liver fibrosis score. Image analysis showed that CAR reduced the liver collagen content (Table 2) and significantly ameliorated the histological structure of the liver compared with non-treated fibrotic rats, showing no signs of steatosis, fibrosis

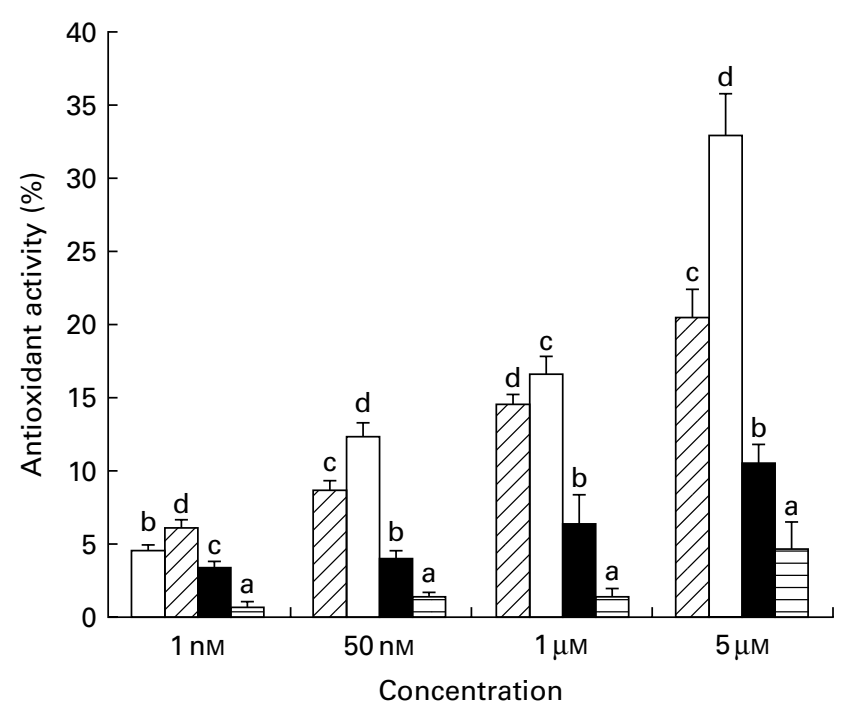

Fig. 1. Antioxidant activity of probucol $(\square), \beta$-caryophyllene ( $\square)$, $\alpha$-tocopherol $(\square)$ and $\alpha$-humulene $(\boxminus)$ on the $\mathrm{Fe}^{2+} /$ ascorbate-induced lipid peroxidation of liver microsomes, expressed as the percentage inhibition of the production of thiobarbituric acid-reactive substances. Values are means, with their standard errors represented by vertical bars $(n 6) .^{\text {a,b,c,d }}$ Mean values with unlike letters were significantly different $(P<0.05)$. 
Table 1. Scavenging effect of probucol, $\alpha$-tocopherol and $\beta$-caryophyllene (CAR) at different concentrations (Mean values with their standard errors, $n 6$ )

\begin{tabular}{|c|c|c|c|c|c|c|c|}
\hline & \multirow[b]{2}{*}{ Concentration $(\mu \mathrm{M})$} & \multicolumn{2}{|c|}{ Probucol } & \multicolumn{2}{|c|}{$\alpha$-Tocopherol } & \multicolumn{2}{|c|}{ CAR } \\
\hline & & Mean & SEM & Mean & SEM & Mean & SEM \\
\hline $\mathrm{IC}_{50} \mathrm{DPPH}(\mu \mathrm{g} / \mathrm{ml})$ & - & $34.0^{\mathrm{b}}$ & 4.7 & $1 \cdot 8^{\mathrm{a}}$ & 0.2 & $132 \cdot 0^{\mathrm{C}}$ & 9.9 \\
\hline \multirow{2}{*}{$\mathrm{OH}^{\circ}$-scavenging activity (\%) } & 1 & 47.8 & $2 \cdot 7$ & 48.0 & 2.5 & $53 \cdot 8$ & 2.9 \\
\hline & 10 & 53.1 & 3.4 & $50 \cdot 0$ & $3 \cdot 1$ & $56 \cdot 8$ & 4.5 \\
\hline \multirow{4}{*}{$\mathrm{O}_{2}^{-}$-scavenging activity (\%) } & 0.1 & $23 \cdot 2^{b}$ & 0.4 & $21 \cdot 6^{a, b}$ & 0.5 & $20 \cdot 1^{a}$ & 0.3 \\
\hline & 1 & $74.8^{a}$ & 1.4 & $86 \cdot 8^{\mathrm{b}}$ & 1.3 & $91.5^{\mathrm{c}}$ & 1.2 \\
\hline & 5 & $93 \cdot 8^{\mathrm{a}}$ & $3 \cdot 1$ & $133.8^{b}$ & $5 \cdot 2$ & $131 \cdot 2^{b}$ & 3.4 \\
\hline & 10 & $154 \cdot 0^{\mathrm{a}}$ & 4.9 & $180 \cdot 8^{b}$ & $7 \cdot 0$ & $205 \cdot 9^{c}$ & $6 \cdot 2$ \\
\hline \multirow{4}{*}{ XO inhibition (\%) } & 0.1 & $44.5^{\mathrm{a}}$ & 1.7 & $58 \cdot 2^{b}$ & 1.2 & $63 \cdot 6^{\mathrm{b}}$ & 1.4 \\
\hline & 1 & $54 \cdot 4^{\mathrm{a}}$ & 1.4 & $72 \cdot 1^{\mathrm{b}}$ & $2 \cdot 2$ & $79 \cdot 5^{\mathrm{b}}$ & $1 \cdot 8$ \\
\hline & 5 & $57.0^{\mathrm{a}}$ & $2 \cdot 1$ & $79 \cdot 4^{\mathrm{b}}$ & $2 \cdot 7$ & $82 \cdot 0^{\mathrm{b}}$ & 2.5 \\
\hline & 10 & $59 \cdot 3^{a}$ & 2.3 & $80 \cdot 1^{b}$ & $2 \cdot 2$ & $84 \cdot 2^{b}$ & 2.5 \\
\hline LPO inhibition (\%) & 0.1 & $12 \cdot 9^{\mathrm{a}}$ & 0.4 & $39.5^{\mathrm{C}}$ & 0.7 & $27 \cdot 9^{b}$ & 0.5 \\
\hline
\end{tabular}

$\mathrm{IC}_{50}$, half-maximal inhibitory concentration; DPPH, 1,1-diphenyl-2-picrylhydrazyl radical; XO, xanthine oxidase; LPO, 5-lipoxygenase. ${ }^{\mathrm{a}, \mathrm{b}, \mathrm{c}}$ Mean values within a row with unlike superscript letters were significantly different $(P<0.05)$.

or hepatocyte necrosis (Fig. 2). Timp1, Tgfb1 and Col1a1 gene expressions were higher in the liver of $\mathrm{CCl}_{4}$-treated rats than in the liver of normal rats (Fig. 3). Furthermore, the CAR treatment significantly attenuated the $\mathrm{CCl}_{4}$-induced up-regulation of both genes. Hence, it was demonstrated that CAR protects the rat liver from $\mathrm{CCl}_{4}$-induced injury by reducing fibrosis and down-regulating Timp1, Tgfb1 and Col1a1 gene expressions.

\section{$\beta$-Caryophyllene protects CFSC-2G hepatic stellate cells against free radical damage}

The next step was to assess whether CAR protected hepatic CFSC-2G cells against oxidative damage induced by a $\mathrm{Fe}^{2+}$ $\mathrm{H}_{2} \mathrm{O}_{2}$ solution. The degree of cell damage was measured with Trypan blue exclusion assay, intracellular TBARS products and enzymatic markers, present in cell media due to cytoplasmic leakage. Oxidative damage reduced cell viability to $93.3 \%$ and increased intracellular TBARS products to $3.72 \mathrm{nmol} / \mathrm{mg}$ protein (Table 3 ). LDH, alkaline phosphatase, $\gamma$-glutamyltranspeptidase, ALAT and ASAT activities were increased in cell media due to free radical-induced cell damage. The CAR treatment increased cell viability and reduced enzyme activities and TBARS production. The present results indicate that CAR effectively protected CFSC-2G cells from free radical-induced cell damage.

\section{$\beta$-Caryophyllene reduces oxidative stress-induced activation of CFSC-2G hepatic stellate cells}

HSC play a key role in response to liver injury. Oxidative stress activates HSC that become the main source of the post-injury extracellular matrix deposit that results in liver fibrosis. An experiment was designed to confirm that CAR reduces HSC activation by reactive oxygen species and down-regulates the mRNA of the Timp1 and Colla1 genes. Exposure of CFSC-2G cells to oxidative damage up-regulated 2.06-fold the expression of the Col1a1 gene and 3·12-fold that of the Timp1 gene in comparison with untreated cells (Fig. 4). The addition of CAR significantly reduced the expression of Col1a1 and Timp1 in a dose-dependent manner.

\section{Discussion}

CAR is a natural volatile bicyclic sesquiterpene that is present in numerous herbs, spices and foods. CAR can be isolated from cinnamon, clove oil and copaiba balsam, all of which have been used as natural remedies. Because of its weak

Table 2. Serum biochemistry of healthy rats (control), carbon tetrachloride-treated rats $\left(\mathrm{CCl}_{4}\right)$ and carbon tetrachloride-treated rats receiving $\beta$-caryophyllene (CAR) at different concentrations

(Mean values with their standard errors, $n 6$ )

\begin{tabular}{|c|c|c|c|c|c|c|c|c|c|c|}
\hline & \multicolumn{2}{|c|}{ Control } & \multicolumn{2}{|c|}{$\mathrm{CCl}_{4}$} & \multicolumn{2}{|c|}{$\mathrm{CAR} 2 \mathrm{mg} / \mathrm{kg}$} & \multicolumn{2}{|c|}{ CAR $20 \mathrm{mg} / \mathrm{kg}$} & \multicolumn{2}{|c|}{ CAR $200 \mathrm{mg} / \mathrm{kg}$} \\
\hline & Mean & SEM & Mean & SEM & Mean & SEM & Mean & SEM & Mean & SEM \\
\hline ASAT (U/I) & $64 \cdot 0^{\mathrm{a}}$ & $5 \cdot 9$ & $379 \cdot 3^{b}$ & $12 \cdot 9$ & $269 \cdot 3^{b}$ & $15 \cdot 1$ & $275 \cdot 3^{b}$ & $14 \cdot 7$ & $292 \cdot 1^{b}$ & $16 \cdot 9$ \\
\hline ALAT (U/I) & $20 \cdot 5^{a}$ & $3 \cdot 8$ & $145 \cdot 7^{b}$ & 7.9 & $61 \cdot 2^{\mathrm{b}}$ & 3.5 & $69 \cdot 4^{b}$ & $3 \cdot 8$ & $72 \cdot 3^{b}$ & $3 \cdot 2$ \\
\hline $\mathrm{LDH}(\mathrm{U} / \mathrm{l})$ & $385 \cdot 0^{\mathrm{a}}$ & $12 \cdot 7$ & $1951 \cdot 8^{b}$ & $35 \cdot 3$ & $1109 \cdot 5^{\mathrm{b}}$ & $27 \cdot 5$ & $912 \cdot 7^{\mathrm{b}}$ & 32.4 & $732 \cdot 4^{b}$ & $42 \cdot 8$ \\
\hline $\mathrm{AP}(\mathrm{U} / \mathrm{l})$ & $165 \cdot 3^{a}$ & $19 \cdot 9$ & $361 \cdot 0^{\mathrm{b}}$ & $32 \cdot 3$ & $320 \cdot 2^{b}$ & $25 \cdot 4$ & $300 \cdot 6^{b}$ & 28.9 & $305 \cdot 1^{b}$ & $32 \cdot 1$ \\
\hline BRB (mg/l) & $6 \cdot 5^{\mathrm{a}}$ & $1 \cdot 2$ & $29 \cdot 3^{c}$ & $3 \cdot 1$ & $12 \cdot 1^{\mathrm{b}}$ & $1 \cdot 7$ & $8 \cdot 7^{\mathrm{b}}$ & $1 \cdot 1$ & $9 \cdot 0^{\mathrm{b}}$ & $1 \cdot 0$ \\
\hline Total protein $(\mathrm{mg} / \mathrm{l})$ & $57 \cdot 0^{\mathrm{b}}$ & $4 \cdot 0$ & $42 \cdot 0^{a}$ & $1 \cdot 0$ & $49 \cdot 0^{b}$ & $3 \cdot 0$ & $53.0^{\mathrm{b}}$ & $2 \cdot 0$ & $56 \cdot 0^{b}$ & $4 \cdot 0$ \\
\hline
\end{tabular}

ASAT, aspartate aminotransferase; ALAT, alanine aminotransferase; LDH, lactate dehydrogenase; AP, alkaline phosphatase; BRB, total bilirubin. ${ }_{a, b, c}$ Mean values within a row with unlike superscript letters were significantly different $(P<0.05)$. 

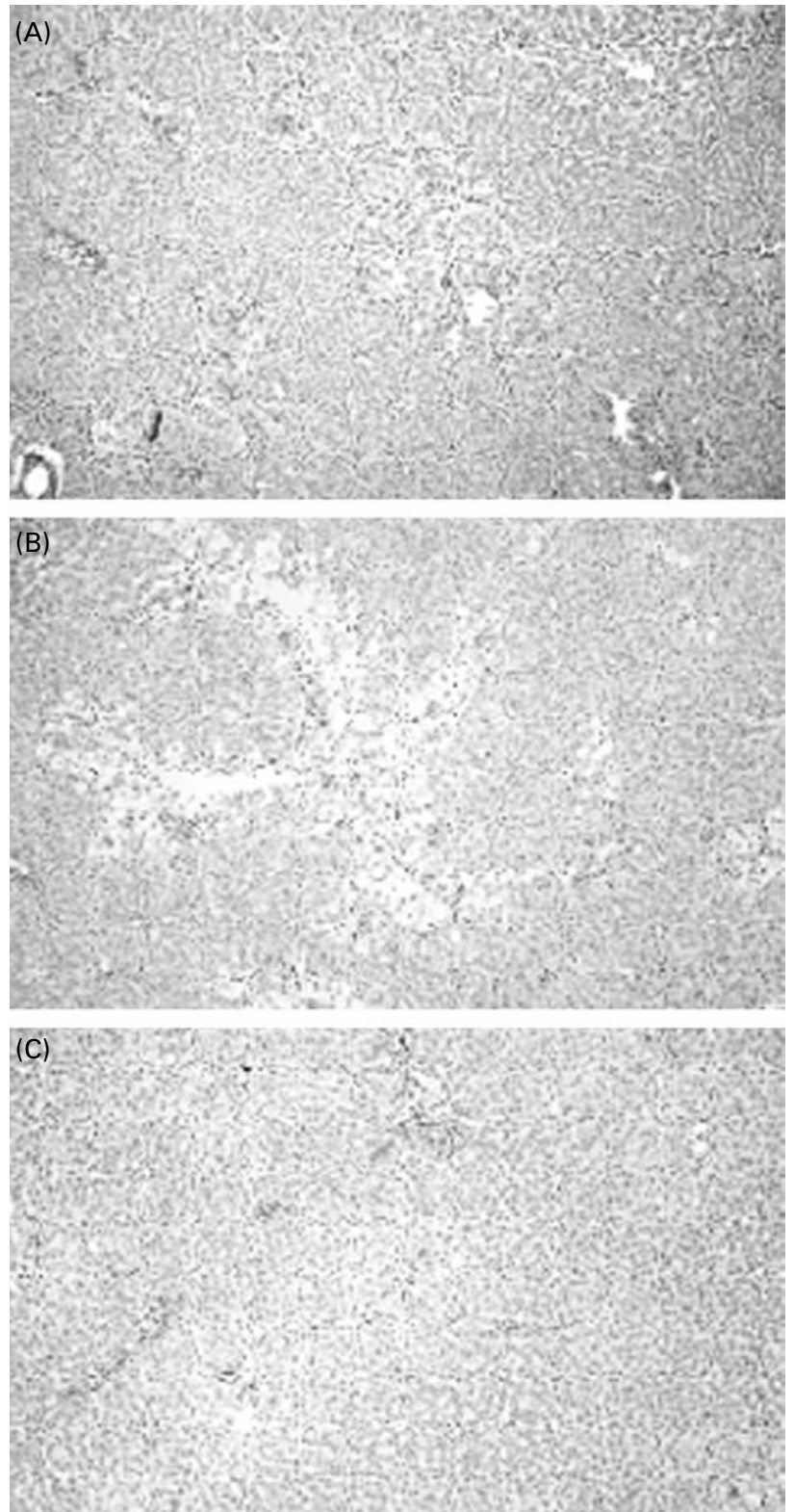

Fig. 2. Protective effect of $\beta$-caryophyllene on carbon tetrachloride-induced liver damage. (A) Sections of the liver of healthy rats, (B) carbon tetrachloride-treated rats and (C) carbon tetrachloride-treated rats that received $2 \mathrm{mg} / \mathrm{kg}$ of $\beta$-caryophyllene. Original magnification, $200 \times$.

aromatic taste, CAR is commercially used as a food additive and in cosmetics. The Flavour and Extract Manufacturers Association granted CAR the status of 'generally recognised as safe', and it has been approved by the US Food and Drug Administration for food use due to its low toxicity ${ }^{(21)}$. CAR has been shown to have anti-microbial ${ }^{(22)}$, antiinflammatory $^{(4)}$, cannabinoid $^{(23)}$, anti-carcinogenic ${ }^{(24)}$ and skin penetration-enhancing properties ${ }^{(25)}$. Our group previously reported the hepatoprotective activity of an ethanol extract of $R$. tomentosus Huberth-Morat and Maire (Lamiaceae) in $\mathrm{CCl}_{4^{-}}$and thioacetamide-treated cirrhotic rats ${ }^{(7,8)}$. The composition of the ethanol extract was unclear, but it contained most of the volatile terpenes present in its essential oil, of which CAR is an important component ${ }^{(9)}$.
Lipid peroxidation of cell membranes is the main damage caused by reactive oxygen species in cells. It is initiated by hydroxyl radicals and superoxide anions, leading to the formation of peroxyl radicals that ultimately propagate the chain reaction in lipids. In a first step, we explored the antioxidant activities of several constituents of Rosmarinus spp. essential oil. CAR was the only terpene to show a strong inhibitory effect on in vitro lipid peroxidation (Fig. 1). This inhibition was significantly higher than that of $\alpha$-humulene, $\alpha$-tocopherol or probucol. Given that different radical species initiate lipid peroxidation, we further tested the free radical-scavenging activity of CAR (Table 1). According to the present results, CAR showed scavenging activity against hydroxyl radical (Fenton reaction) and superoxide anion (riboflavin assay) in a concentration-dependent manner (Table 1). Its scavenging capacity was significantly higher than that of probucol. The scavenging properties of $\alpha$-tocopherol and CAR were similar. However, both sesquiterpenes, CAR and $\alpha$-humulene failed to scavenge DPPH, a molecule that contains a stable free radical. This result may explain why CAR has not been previously reported as an antioxidant, since the DPPH test is the most widely used technique to characterise the anti-radical capacity of compounds. Interestingly, CAR was able to markedly reduce the in vitro formation of lipid peroxides by 5-lipoxygenase. Taken together, our data indicated that CAR is a highly effective chain-breaking antioxidant in in vitro lipid peroxidation and possesses greater scavenging activities against reactive oxygen species than against stable organic radicals.

Based on these results, we hypothesised that CAR may protect from oxidative stress-induced injury. Hepatic fibrosis caused by $\mathrm{CCl}_{4}$ has been extensively used in experimental rat models. Hepatic responses in rats to acute $\mathrm{CCl}_{4}$ administration are shown to be similar to human hepatic fibrosis ${ }^{(26)}$. $\mathrm{CCl}_{4}$ metabolism in the liver stimulates lipid peroxidation

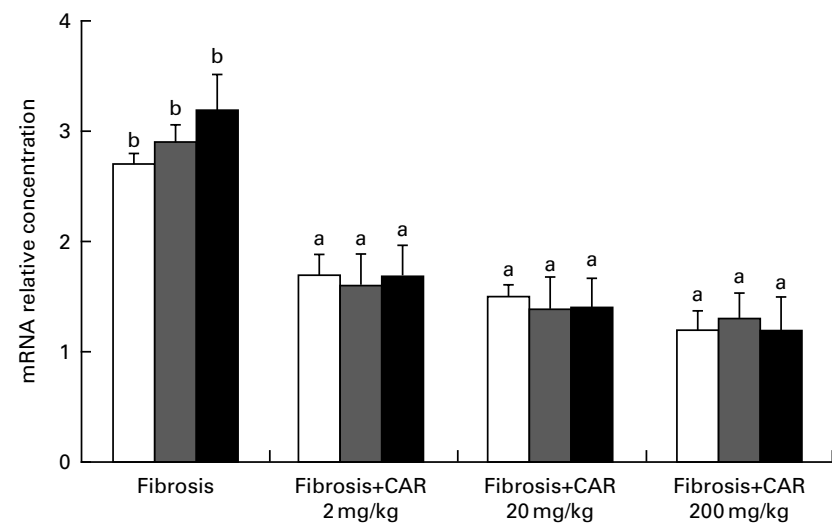

Fig. 3. Effect of $\beta$-caryophyllene (CAR) on the hepatic mRNA expression of the type I collagen $\alpha 1$ (Col1a1, $\square$ ), transforming growth factor- $\beta 1$ (Tgfb1, $\square$ ) and tissue inhibitor of metalloproteinase-1 (Timp1, $\square$ ) genes in carbon tetrachloride-induced liver fibrosis. Rats received different concentrations of CAR. Data are values of mRNA expression relative to glyceraldehyde-3-phosphate dehydrogenase (Gapdh). Values are means, with their standard errors represented by vertical bars $(n 3)$. ${ }^{\mathrm{a}, \mathrm{b}}$ Mean values with unlike letters were significantly different $(P<0.05)$. 
Table 3. Protection of $\beta$-caryophyllene (CAR) against oxidative stress-induced damage to CFS-2G hepatic stellate cells

(Mean values with their standard errors, $n 6$ )

\begin{tabular}{|c|c|c|c|c|c|c|c|c|c|c|c|c|}
\hline & \multicolumn{2}{|c|}{ Control ${ }^{\star}$} & \multicolumn{2}{|c|}{$\begin{array}{l}\text { Oxidative } \\
\text { damage† }\end{array}$} & \multicolumn{2}{|c|}{$\begin{array}{l}\alpha \text {-Tocopherol } \\
(1 \mu \mathrm{M})\end{array}$} & \multicolumn{2}{|c|}{$\begin{array}{l}\alpha \text {-Tocopherol } \\
(10 \mu \mathrm{M})\end{array}$} & \multicolumn{2}{|c|}{ CAR $(1 \mu \mathrm{M})$} & \multicolumn{2}{|c|}{ CAR $(10 \mu \mathrm{M})$} \\
\hline & Mean & SEM & Mean & SEM & Mean & SEM & Mean & SEM & Mean & SEM & Mean & SEM \\
\hline TBARS (nmol/mg protein) & $0.62^{\mathrm{a}}$ & 0.07 & $3.72^{\mathrm{c}}$ & $1 \cdot 2$ & $0.92^{b}$ & 0.09 & $0.75^{\mathrm{b}}$ & 0.08 & $0.52^{\mathrm{a}}$ & 0.08 & $0.50^{\mathrm{a}}$ & 0.05 \\
\hline Cell viability (\%) & - & - & $93 \cdot 3^{a}$ & 2.5 & $99 \cdot 1^{a}$ & $2 \cdot 4$ & $99 \cdot 5^{\mathrm{a}}$ & $2 \cdot 1$ & $98 \cdot 8^{\mathrm{a}}$ & $2 \cdot 7$ & $99 \cdot 3^{a}$ & $2 \cdot 2$ \\
\hline $\mathrm{LDH}(\mathrm{U} / \mathrm{l})$ & $12 \cdot 5^{\mathrm{a}}$ & $1 \cdot 1$ & $23 \cdot 5^{\mathrm{b}}$ & $2 \cdot 6$ & $11.5^{a}$ & 1.0 & $11.0^{\mathrm{a}}$ & $1 \cdot 2$ & $13 \cdot 5^{\mathrm{a}}$ & $1 \cdot 1$ & $12 \cdot 8^{\mathrm{a}}$ & $1 \cdot 2$ \\
\hline ASAT (U/I) & $21 \cdot 0^{\mathrm{a}}$ & 1.4 & $33.9^{\mathrm{c}}$ & $2 \cdot 3$ & $25 \cdot 2^{b}$ & 1.5 & $20 \cdot 8^{a}$ & 1.3 & $25 \cdot 8^{\mathrm{b}}$ & 1.3 & $21.8^{\mathrm{a}}$ & 1.0 \\
\hline ALAT (U/I) & $24 \cdot 7^{c}$ & 0.8 & $34 \cdot 8^{d}$ & 1.6 & $23 \cdot 8^{b, c}$ & 0.4 & $23 \cdot 1^{b}$ & 0.4 & $22 \cdot 0^{a}$ & 0.3 & $23 \cdot 8^{b, c}$ & 0.5 \\
\hline $\mathrm{AP}(\mathrm{U} / \mathrm{l})$ & $28 \cdot 8^{\mathrm{a}}$ & 0.4 & $40 \cdot 2^{c}$ & 0.5 & $30 \cdot 7^{b}$ & 0.3 & $28 \cdot 9^{a}$ & 0.5 & $31 \cdot 8^{\mathrm{b}}$ & 0.4 & $29 \cdot 7^{a, b}$ & 0.3 \\
\hline$\gamma$-GT (U/I) & $13 \cdot 2^{a}$ & 0.3 & $30 \cdot 3^{d}$ & 0.5 & $18 \cdot 2^{\mathrm{c}}$ & 0.3 & $15 \cdot 3^{\mathrm{b}}$ & 0.4 & $18 \cdot 7^{\mathrm{c}}$ & 0.5 & $16 \cdot 7^{\mathrm{b}}$ & 0.4 \\
\hline
\end{tabular}

TBARS, thiobarbituric acid-reactive substances; LDH, lactate dehydrogenase; ASAT, aspartate aminotransferase; ALAT, alanine aminotransferase; AP, alkaline phosphatase; $\gamma$-GT, $\gamma$-glutamyl transpeptidase.

a,b,c,d Mean values within a row with unlike superscript letters were significantly different $(P<0.05)$.

* Control cells received vehicle alone (ethanol).

† Oxidative damage was induced in cells by addition of an $\mathrm{Fe}^{2+} /$ ascorbate $50 \mu \mathrm{m} / 200 \mu \mathrm{m}$ solution. Cells were incubated with $\alpha$-tocopherol or CAR before oxidative damage.

and the production of free radicals ${ }^{(27)}$, causing necrosis of hepatocytes, inducing inflammation and further promoting the progression of hepatic fibrogenesis. In this mechanism, activation of Kupffer cells, where 5-lipoxygenase is basically expressed in the liver ${ }^{(28)}$, and the subsequent release of proinflammatory mediators are an early step in the pathogenesis of fibrosis. Since CAR inhibited in vitro lipid peroxidation and 5-lipoxygenase activity, we tested its effect on $\mathrm{CCl}_{4}$ liver toxicity. Acute poisoning with $\mathrm{CCl}_{4}$ increased bilirubin concentration and ALAT, ASAT, alkaline phosphatase and LDH activities in rat plasma, decreased in plasma total protein values, and produced severe fibrosis of hepatocyte necrosis, inflammatory cell infiltration, fibrosis and microvesicular steatosis (Table 2; Fig. 2). CAR demonstrated hepatoprotective activity, markedly restoring the values of plasma markers of liver damage and reducing the degree of steatosis and the extent of liver fibrosis.

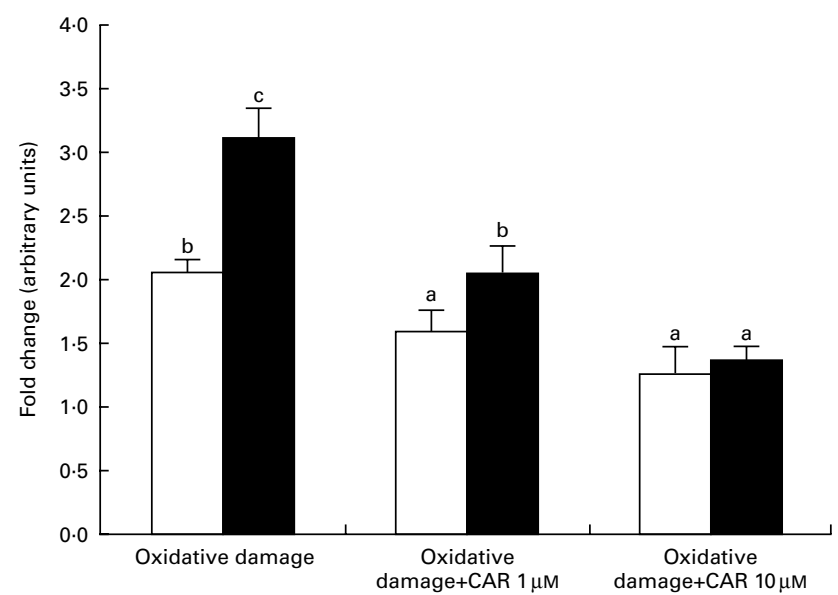

Fig. 4. $\beta$-Caryophyllene (CAR) reduces oxidative stress-induced activation of CFS-2G hepatic stellate cells. Data of mRNA expression of the type I collagen $\alpha 1$ (Col1a1, $\square$ ) and tissue inhibitor of metalloproteinase-1 (Timp1, 口) genes relative to glyceraldehyde-3-phosphate dehydrogenase (Gapdh) are presented. Oxidative damage was induced with a $\mathrm{Fe}^{2+} /$ ascorbate $50 \mu \mathrm{M} / 200 \mu \mathrm{M}$ solution. Cells were treated with CAR at the indicated concentrations before oxidative damage. Values are means, with their standard errors represented by vertical bars $(n 3) .{ }^{a, b, c}$ Mean values with unlike letters were significantly different $(P<0.05)$.
It is the exuberant deposition of matrix proteins after injury that causes liver fibrosis and eventually cirrhosis. The mechanism of hepatic fibrogenesis is related not only to an enhanced matrix biosynthesis but also to an inhibition of matrix breakdown ${ }^{(29)}$. In human subjects, chronic alcohol-induced damage to the liver significantly diminishes collagenolytic activity in parallel with a dramatic rise in TIMP-1 and TGF- $\beta$ levels ${ }^{(30)}$. In the present experiment, $\mathrm{CCl}_{4}$ poisoning markedly increased the expression of the Col1a1, Tgfb1 and Timp1 genes. Protection by $\mathrm{CAR}$ against $\mathrm{CCl}_{4}$-induced liver fibrosis was associated with a reduction in liver collagen content, the histology score and the expression of the Col1a1, Tgfb1 and Timp1 genes (Fig. 3). These results indicate that CAR is a hepatoprotective compound that down-regulates the expression of profibrotic genes and reduces hepatic histological lesions.

Oxidative stress promotes the activation of HSC that are the main source of the post-injury extracellular matrix deposit resulting in liver fibrosis. Oxidative stress promotes HSC proliferation and up-regulates Col1a1, Tgfb1 and Timp1 gene expressions in $\mathrm{HSC}^{(31)}$, and this process is modulated by antioxidants ${ }^{(32,33)}$. Based on our previous results, we hypothesised that CAR may prevent liver fibrosis by reducing HSC activation. An experiment was designed to test whether CAR inhibited oxidative stress-mediated HSC activation. Reactive oxygen species have been shown to disrupt cell membrane integrity due to the oxidation of PUFA, which causes the spillage of cytoplasmic enzymes. As expected, exposure of CFCS-2G cells to a pro-oxidant solution reduced cell viability and increased LDH, ASAT, ALAT, alkaline phosphatase and $\gamma$-glutamyltranspeptidase leakage, and malonaldehyde concentration in culture media (Table 3). Oxidative damage up-regulated the expression of the Col1a1 and Timp1 genes in CFSC-2G cells (Fig. 4). Pretreatment of CFSC-2G cells with CAR significantly reduced oxidative stress-induced damage in a dose-dependent manner. The main result was the down-regulation of Col1a1 and Timp1 gene transcription exerted by CAR. In keeping with our hypothesis, the present results confirmed that CAR is an effective inhibitor of cell damage induced by reactive oxygen species and can prevent 
oxidative stress-mediated up-regulation of the Col1a1 and Timp1 genes in HSC.

In summary, we provide the first demonstration that CAR, a compound present in numerous plants and foods, is an effective inhibitor of lipid peroxidation, probably due to its free radical-scavenging activity against hydroxyl radicals, superoxide anions and lipid peroxides. Our data provide novel insights into the mechanisms of CAR in the prevention of liver fibrosis that may be related at least in part to its antioxidant activity. Since CAR is present in numerous plants, spices and foods, further experiments are needed to consolidate the potential application of CAR as a bioactive natural compound that reduces oxidative stress-mediated injury in human diseases.

\section{Acknowledgements}

None of the authors had a commercial interest, financial interest and/or other relationships with pharmaceutical, laboratory supplies or medical companies. The authors are grateful to the Spanish Ministry of Public Health for providing funds during the tenure of research. A. S. was involved in the data collection, data analysis, data interpretation, literature search and manuscript preparation. M. A. C., J. M. V., T. M. and M. I. T. were involved in the data collection, data analysis and data interpretation. A. S., M. J. F. and A. G. were involved in the study design and data interpretation.

\section{References}

1. Pomerleau J, Lock K, Knai C, et al. (2005) Interventions designed to increase adult fruit and vegetable intake can be effective: a systematic review of the literature. $J$ Nutr 135, 2486-2495.

2. Liu RH (2004) Potential synergy of phytochemicals in cancer prevention: mechanism of action. J Nutr 12, S3479-S3485.

3. Goff SA \& Klee H (2006) Plant volatile compounds: sensory cues for health and nutritional value? Science $\mathbf{3 1 1}$, 815-819

4. Aggarwal RB \& Rangari VD (2003) Phytochemical investigation and evaluation of anti-inflammatory and anti-arthritic activities of essential oil of Strobilanthus ixiocephala Benth. Indian J Exp Biol 41, 890-894.

5. Tirapelli CR, Ambrosio SR, da Costa FB, et al. (2008) Diterpenes: a therapeutic promise for cardiovascular diseases. Recent Patents Cardiovasc Drug Discov 3, 1-8.

6. Sethi G, Ahn KS, Pandey MK, et al. (2007) Celastrol, a novel triterpene, potentiates TNF-induced apoptosis and suppresses invasion of tumor cells by inhibiting NF-kappaBregulated gene products and TAK1-mediated NF-kappaB activation. Blood 109, 2727-2735.

7. Galisteo M, Suárez A, del Pilar Montilla M, et al. (2000) Antihepatotoxic activity of Rosmarinus tomentosus in a model of acute hepatic damage induced by thioacetamide. Phytother Res 14, 522-526.

8. Galisteo M, Suárez A, Montilla MP, et al. (2006) Protective effects of Rosmarinus tomentosus ethanol extract on thioacetamide-induced liver cirrhosis in rats. Phytomedicine 13, 101-108.

9. del Baño MJ, Lorente J, Castillo J, et al. (2003) Phenolic diterpenes, flavones, and rosmarinic acid distribution during the development of leaves, flowers, stems, and roots of
Rosmarinus officinalis. Antioxidant activity. I Agric Food Chem 51, 4247-4253.

10. Greenwel P, Rubin J, Schartz M, et al. (1993) Liver fat-storing cell clones obtained from a $\mathrm{CCl}_{4}$-cirrhotic rat are heterogeneous with regard to proliferation, extracellular matrix components, interleukin-6 and connexin 43. Lab Invest 69, 210-216.

11. Cho JY, Chang HJ, Lee SK, et al. (2007) Amelioration of dextran sulfate sodium-induced colitis in mice by oral administration of CAR, a sesquiterpene. Life Sci 80, 932-939.

12. Zhen MC, Wang Q, Huang XH, et al. (2007) Green tea polyphenol epigallocatechin-3-gallate inhibits oxidative damage and preventive effects on carbon tetrachloride-induced fibrosis. J Nutr Biochem 18, 795-805.

13. Pérez MJ, Suárez A, Gómez-Capilla JA, et al. (2002) Dietary nucleotide supplementation reduces thioacetamide-induced liver fibrosis in rats. J Nutr 132, 652-657.

14. Mensor LL, Menezes FS, Leitão GG, et al. (2001) Screening of Brazilian plant extracts for antioxidant activity by the use of DPPH free radical method. Phytother Res 15, 127-130.

15. Xun S, Jingdong T, Zhaonan Z, et al. (1991) Chemiluminescence study on the peroxidation of linoleic acid initiated by the reaction of ferrous iron with hydrogen peroxide. Biophys Chem 40, 161-167.

16. Bridges AB, Scott NA \& Belch JJ (1991) Probucol, a superoxide free radical scavenger in vitro. Atherosclerosis 89 , $263-265$.

17. Yin MC \& Chan KC (2007) Nonenzymatic antioxidative and antiglycative effects of oleanolic acid and ursolic acid. J Agric Food Chem 55, 7177-7181.

18. Liu YJ \& Pan BS (2004) Inhibition of fish gill lipoxygenase and blood thinning effects of green tea extract. J Agric Food Chem 52, 4860-4864.

19. Suárez A, Ramírez-Tortosa M, Gil A, et al. (1999) Addition of vitamin E to long-chain polyunsaturated fatty acid-enriched diets protects neonatal tissue lipids against peroxidation in rats. Eur J Nutr 38, 169-176.

20. Bradford MM (1976) A rapid and sensitive method for the quantitation of microgram quantities of protein utilizing the principle of protein-dye binding. Anal Biochem $\mathbf{7 2}$, $248-254$.

21. Adams TB, Gavin CL, McGowen MM, et al. (2011) The FEMA GRAS assessment of aliphatic and aromatic terpene hydrocarbons used as flavor ingredients. Food Chem Toxicol 49 , 2471-2494.

22. Sabulal B, Dan M, J AJ, et al. (2006) Caryophyllene-rich rhizome oil of Zingiber nimmonii from South India: chemical characterization and antimicrobial activity. Phytochemistry 67, 2469-2473.

23. Gertsch J, Leonti M, Raduner S, et al. (2008) Beta-caryophyllene is a dietary cannabinoid. PNAS 105, 9099-9104.

24. Legault J \& Pichette A (2007) Potentiating effect of betacaryophyllene on anticancer activity of alpha-humulene, isocaryophyllene and paclitaxel. J Pharm Pharmacol 59, 1643-1647.

25. Cornwell PA \& Barry BW (1994) Sesquiterpene components of volatile oils as skin penetration enhancers for the hydrophilic permeant 5-fluorouracil. J Pharm Pharmacol 46, 261-269.

26. Pérez Tamayo R (1983) Is cirrhosis of the liver experimentally produced by $\mathrm{CCl}_{4}$ and adequate model of human cirrhosis? Hepatology 3, 112-120.

27. Basu S (2003) Carbon tetrachloride-induced lipid peroxidation: eicosanoid formation and their regulation by antioxidant nutrients. Toxicology 189, 113-127. 
28. Titos E, Clària J, Planagumà A, et al. (2003) Inhibition of 5-lipoxygenase induces cell growth arrest and apoptosis in rat Kupffer cells: implications for liver fibrosis. FASEB J 1, 1745-1747.

29. Friedman SL (2008) Mechanisms of hepatic fibrogenesis. Gastroenterology 134, 1655-1669.

30. Iredale JP, Benyon RC, Arthur MJ, et al. (1996) Tissue inhibitor of metalloproteinase- 1 messenger RNA expression is enhanced relative to interstitial collagenase messenger RNA in experimental liver injury and fibrosis. Hepatology 24, 176-184.
31. Galli A, Svegliati-Baroni G, Ceni E, et al. (2005) Oxidative stress stimulates proliferation and invasiveness of hepatic stellate cells via a MMP2-mediated mechanism. Hepatology 41, 1074-1084.

32. Orr JG, Leel V, Cameron GA, et al. (2004) Mechanism of action of the antifibrogenic compound gliotoxin in rat liver cells. Hepatology 40, 232-242.

33. Martucci RB, Ziulkoski AL, Fortuna VA, et al. (2004) Betacarotene storage, conversion to retinoic acid, and induction of the lipocyte phenotype in hepatic stellate cells. J Cell Biochem 92, 414-423. 